\title{
Experimental and Numerical Analysis of Dynamic Properties of Wound and Wrapped Car- bon Composites
}

Petr Kulhavý, Vitezslav Fliegel

Faculty of Mechanical Engineering, Technical University of Liberec. Studentska 2, 46117 Liberec 1, Czech Republic.

E-mail: petrkhy@seznam.cz, vitezslav.fliegel@tul.cz

In the presented work, static and dynamic properties of two, theoretically identical composite rods were studied. Those two kinds of rod were made of pre-impregnated fibers so called "prepregs". The first of them was made by means of wrapping technology, which is simply just helical layering of one wide tape around a rotating mandrel. The weakness of this method is possible using only for straight parts. That is why a simultaneous deposition of several thinner filaments in a form of tape called winding was used in order to optimize this technology also for curved parts with various cross-sections.

The target of this work was to compile numerical model and experimentally compare flexural strength of the theoretically identical wrapped and wound rods in the static three point test.Further small samples cut and extracted from those rods were subjected to dynamic bending loading on Dynamic Mechanical Analyzer, with various loading speed, in order to obtain also the dynamic properties. The found results and conclusions of this article should provide a basic idea how significantly the manufacturing process could affects the microstructure and real mechanical properties of long fibers composite parts.

Keywords: Carbon Composites, Bending Test, DMA, Winding, Wrapping

\section{Introduction}

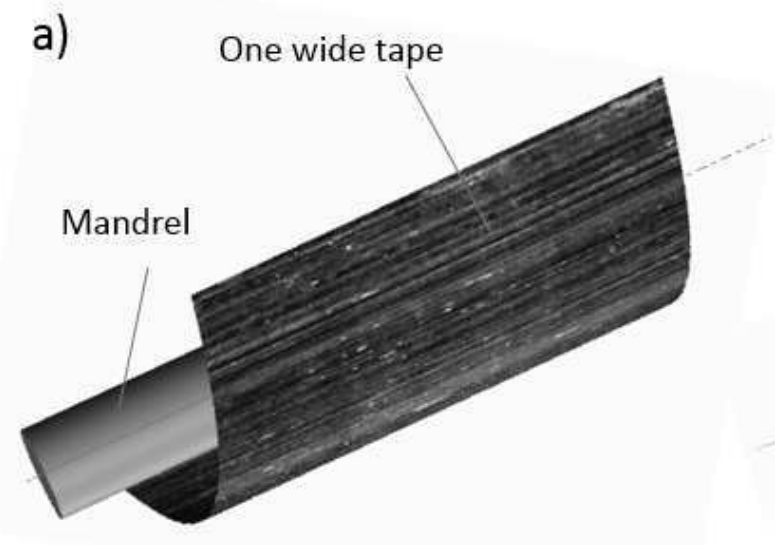

b)

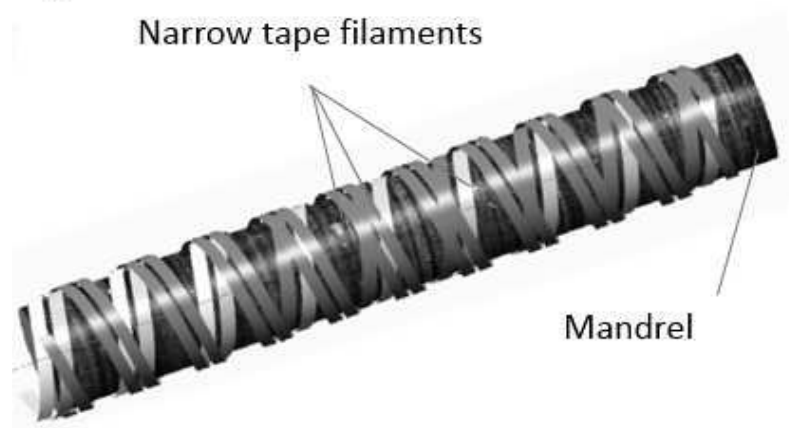

Fig. 1 Principles of the two manufacturing methods a) Wrapping b) Winding

Reduction of expenditure, improving quality and design based on modern CAD technologies are the key points in the future of composite parts. Composite materials are used especially because of their low weight, very high strength and could offer solution also for applications subjected to fatigue loadings. It is possible to reduce the weight of vehicles up to 60 per cent by changing the material from metal to composites [1]. Compared to metallic structures, composites offer some unique engineering properties while presenting interesting but challenging problems for analysts and designers [2]. The most effective replacement of conventional materials by composites is possible with the appropriate combination of manufacturing technologies, stack up of individual layers, dispersion and matrix [3]. Plastics materials reinforced by long fibers are widely used because of their high strength and modulus to density ratio [4]. During the development of composite parts it is necessary to consider lot of aspects (grease, pressure, temperature, imperfect vacuum, varying thickness of plies) and this all could significantly affect the final mechanical parameters. Instead of using classical dry fibres, this work describes using presaturated material so called prepregs [5]. The main benefit of using pre-impregnated fibers instead of filaments is the simplification (or even elimination) of consequent technologies like saturation and curing in form (wen). The aim of this article is to compare static and dynamic behavior in flexural loading of two theoretically identical parts created from preimpregnated fibres by different methods

\section{Materials and methods}

Currently technologies like winding of fibers, tape wrapping, laminating of fabric layers and some other operations are often used for manufacturing the so called advanced composites [6]. Methods based on epicyclic winding or helical wrapping (Fig. 1) are ordinarily used for manufacturing of thin-walled composite parts with circular or oval profiles. Those methods are usually used for so-called "wet" case, when a bundle of dry placed fibers 
is subsequently impregnated with resin. Another way is to use of the pre impregnated materials. Because of their significantly different behavior (sticky, such as double- sided tape), their use is generally limited primarily to manufacturing straight bars.
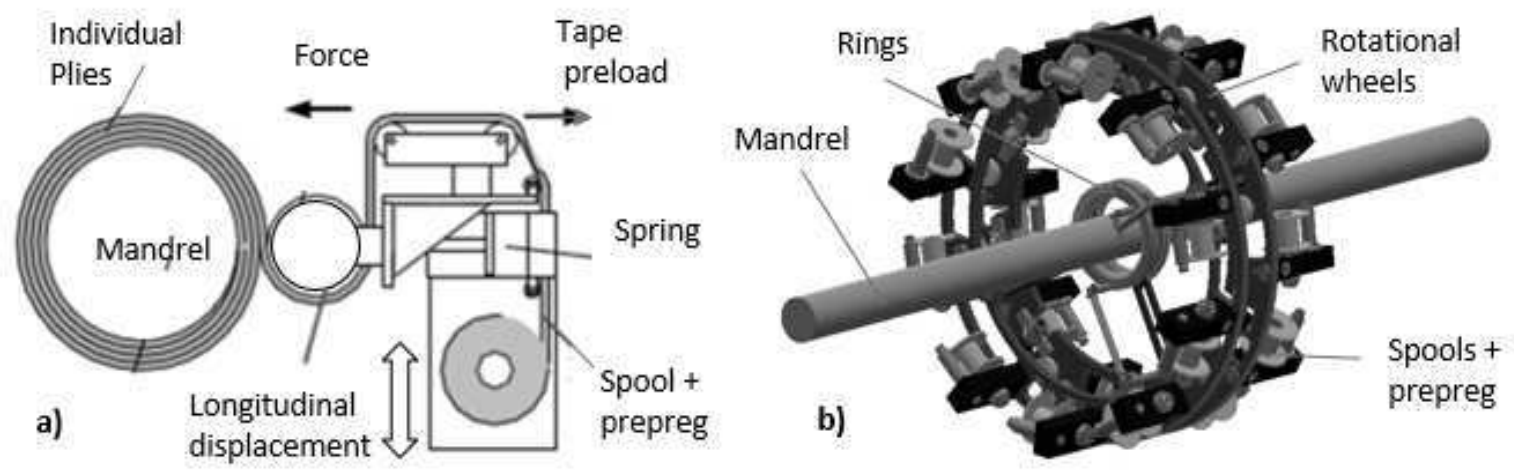

Fig. 2 Scheme of the used manufacturing devices a) Wrapping b) Winding

So one of the used technologies is wrapping. It means helical layering of a wide tape around a straight or eventually conical mandrels (Fig. 2). The second used technology, called winding is a manufacturing process that should keep the fibers continuous and aligned throughout entire part, offers a high degree of automation and relatively high processing speeds [1]. The process of winding fibers is known for a long time, however this technology was concerned especially to field of textile engineering (braiding of ropes, hoses) and parts from some atypical sectors. Nowadays, due to big progress in using advanced composite materials instead of conventional, it is possible to meet the wound parts in many various industries.

The fundamental idea of this method was based on a registered patent [7]. The winding head is formed by a base frame and two rotary wheels, which carry spools with prepreg tapes used for winding. During the process of winding the mandrel is pulled through the center of the rotational head. Compared to a conventional winding of dry fibers there are significantly bigger axial forces caused by the dragging fibers over aluminum rings, some passive resistances and a braking moment in the spool mechanism. Through the fibers, the force comes to the rotary wheel [8]. As mentioned Zhang [9] for ensuring the best properties it is necessary to preload the tape with a quite big and invariable force to improve alignment of individual fibers and to prevent warping and premature sticking of the winded tape.

To assure the most optimal properties of the basic material (Tab. 1) should the semi-finished pre-impregnated fibers come from the manufacturers in frozen state with the individual plies protected by a silicone foil. The tensile strength in the direction perpendicular to the direction of longitudinal fibers is for uni-directional materials even lower than the ultimate strength of the matrix itself, which is caused by the concentration of local stress on their interface [2].

Tab. 1 Material properties of used UD prepreg materials

\begin{tabular}{|c|c|c|c|c|c|}
\hline & E1 [MPa] & E2 $[\mathrm{MPa}]$ & $\rho\left[\mathrm{g} / \mathrm{cm}^{3}\right]$ & $\mu[-]$ & Ductility [\%] \\
\hline UD Prepreg & 91000 & 9000 & 1.9 & 0.25 & $\lim ->0$ \\
\hline
\end{tabular}

With the optimal settings the resulting layer should have the same angle of the fibers, thickness, weight and theoretically almost identical mechanical properties. During the production of the sample, attention was paid to the composition, number of layers, weight and curing parameters those should mutually correspond as much as

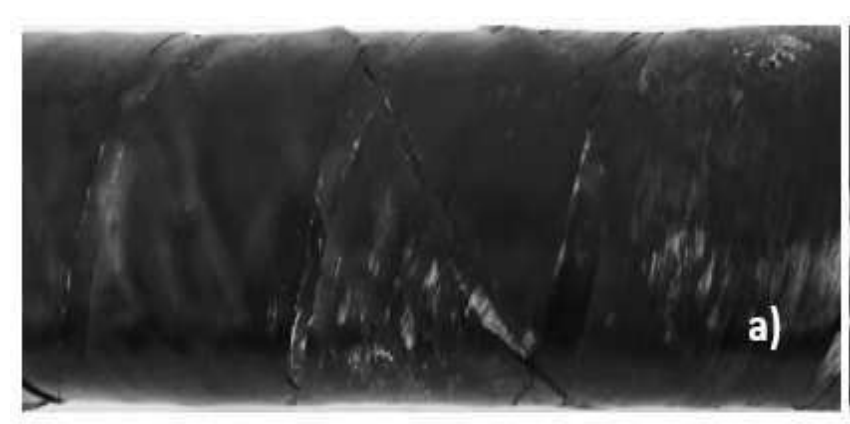

possible. As could be seen in the Fig. 3 it is possible to find first visual differences immediately after the curing. During the winding process arising a lot of seemingly small unimportant defects in the fiber aligning and also sticking of the tape's edges.

Fig. 3 Detail of the surface of the a) Wrapped b) Wound, rods made of prepreg 


\section{Models and experiment}

Prediction of mechanical behavior of composites is a very complex problem, because the process induces whole spectrum of attributes like the fibres orientation, interface properties, cohesive forces and failure criteria. Advanced methods could describe the entire damage process from its initiation to a complete failure of a composite structure [10]. The unanswered question is how accurate the simulation should be to be suitable: the mesh relevance, chosen formulations, failure criteria etc., when we consider the initial error caused by the material model and boundaries.

Because modeling of contact like in our case the between layered shell and solid element is very problematic, It is possible to find various simplifications, e.g.

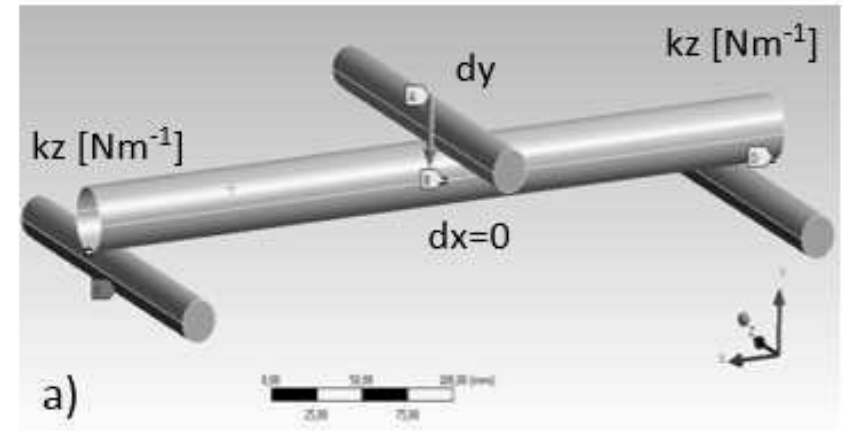

b)

Gruber [11] created and simplified static model of the part alone without using any contact. In our case, the model was solved as a fully contact task, Fig. 4 . The tested part was laid on two fixed supports. For combination of solid and shell elements the pure penalty formulation with nodal-normal detection of integration points was used. The chosen basic normal stiffness $1 \mathrm{e}-002$ could be additionally adjusted by program. Because the fact that in the first time steps before the contact of the solid supports and layered rods arise, the model is unconstrained and it is necessary to add a weak spring. The spring constant could dependent on the loading parameter or a time step of solution, so the spring could have effect only in the really beginning of the simulation [12]. Kirstein [13] even described thesis that during the test could be the boundary of the plate considered to be free as the plate is supported at interior points, and no special treatment is required.

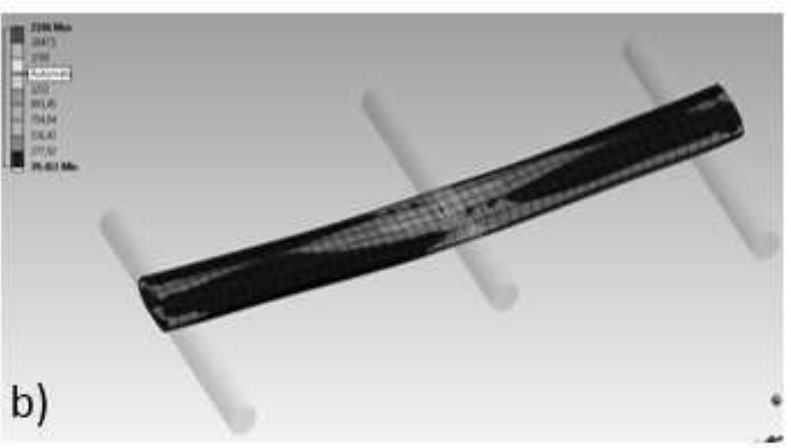

Fig. 4 The numerical model of shell composite bending test a) Scheme with the boundary conditions b) An example of resulting stress in one ply

In our case the problem of numerical simulation of composite materials is an assumption of a homogeneous system with perfectly aligned fibers and their uniform distribution throughout the cross section. This idea is commonly applied to the simulation of laminated and wrapped parts. However, how much the results differ for the winding, (i.e. method with large randomness in the fiber alignment and even overlapping of filaments) is a question for comparing individual results in next chapter.

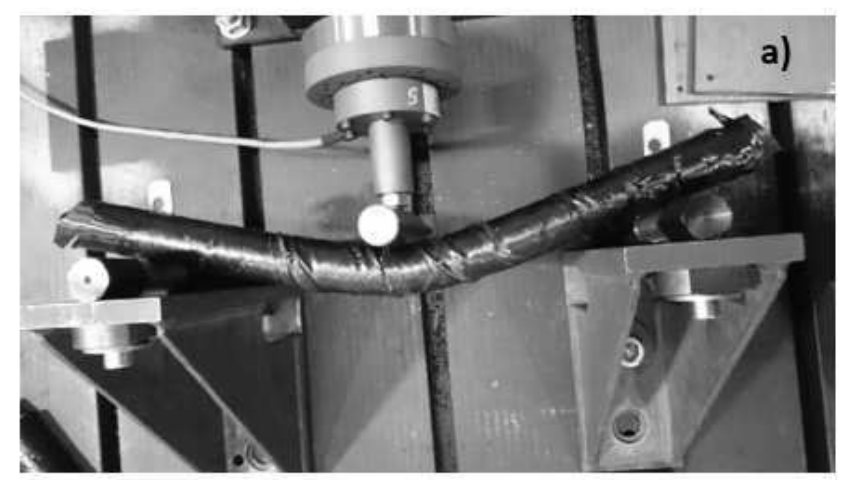

\subsection{Static analysis}

The flexural strength is the maximum stress that material subjected to bending load could resist before failure $[14,15]$. The three point bending test (Fig. 5) has been conducted in the standard, generally valid conditions. The distance $l$ between the cylindrical supports was in our case equal to $380 \mathrm{~mm}$. The applied quasi static loading had increased with step $0.5 \mathrm{~mm} / \mathrm{s}$ until caused the final displacement of used indenter $80 \mathrm{~mm}$.

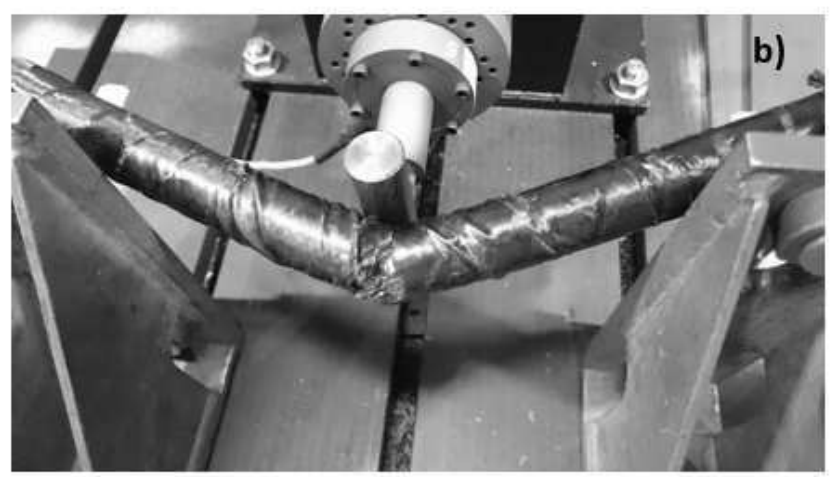

Fig. 5 Three point flexural testing of the a) Wrapped b) Wound rods

\subsection{Dynamic analysis}

Because in the point of view to the real operating conditions of technical devices the basic static test is not sufficient, the samples were tested also on the DMA (dynamic mechanical analyzer, Fig.6). The chosen method was 3 point bending with constant strain and variing frequencies. The dynamic three point loading also with the 4-points bend are probably the most appropriate methods useable for testing layered composites [16,17]. Based on the measurement on DMA the storage and loss modulus 
of the reinforced plastic samples could be determined.

The frequency sweep was performed to evaluate the viscoelastic response of a material by the observed changes in the storage and loss moduli and $\tan \delta$. The loss tangent is the ratio of loss modulus and storage modulus, which indicate the viscosity and elastic properties of material, respectively.

In the DMA experimental device, the amplitude is defined as the maximum amount of oscillatory motion in micrometers (one half peak-to-peak). According to [18] for composite samples the amplitude of 10 to $50 \mu \mathrm{m}$ should yield good results and it is recommended to use "Logarithmically spaced" frequencies (e.g., 10, 5, 2, 1) rather than "linearly spaced" frequencies (e.g., 9, 7, 5, 3, 1).

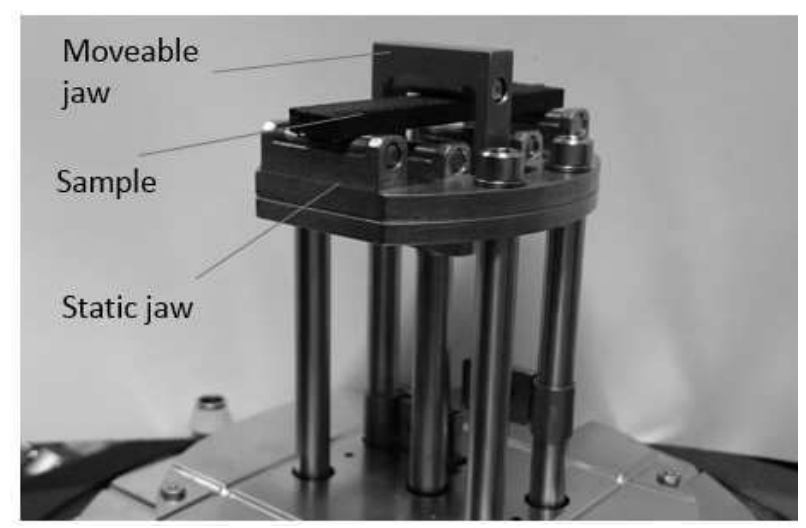

Fig. 6 The dynamic experiment on DMA

The dynamic force is that one required to maintain the programmed amplitude at a certain frequency [dma].
When force track is activated, the static force is no longer constant, but is dependent on the dynamic force. The ratio of static to dynamic force is essentially for the force track. The following formula (1) was used to maintain the ratio between the preload force and dynamic force (force to drive amplitude).

$$
F_{S}=F_{T} \times K \times A
$$

Where the $\mathrm{F}_{\mathrm{s}}$ is the static force $[\mathrm{N}], \mathrm{F}_{\mathrm{T}}$ the force track $[-]$, the $\mathrm{K}$ stiffness $[\mathrm{N} / \mathrm{m}]$ and $\mathrm{A}$ is the amplitude $[\mathrm{m}]$.

\section{Results and discussion}

The resulting flexural strength obtained for the two groups of tested parts were significantly different. For the static test, there are differences not only in the maximum stress level, but also in the value of displacement and also the places of material ruptures looks differently. For the wrapping case there were mainly delamination and the filament ruptures for the winding. The carried model (Fig. 7) was in the beginning (i.e. until $25 \mathrm{~mm}$ of displacement) in a good agreement with the wrapped parts. Then, in the real part some first ruptures and delamination arose, while the force in the model still increased up to the deformation of $35 \mathrm{~mm}$, where was not possible to reach the convergence of solution anymore.

When assessing the second test case, wound parts (not the one wide tape but 10 segments of relatively narrow filaments) the obtained values were significantly different.

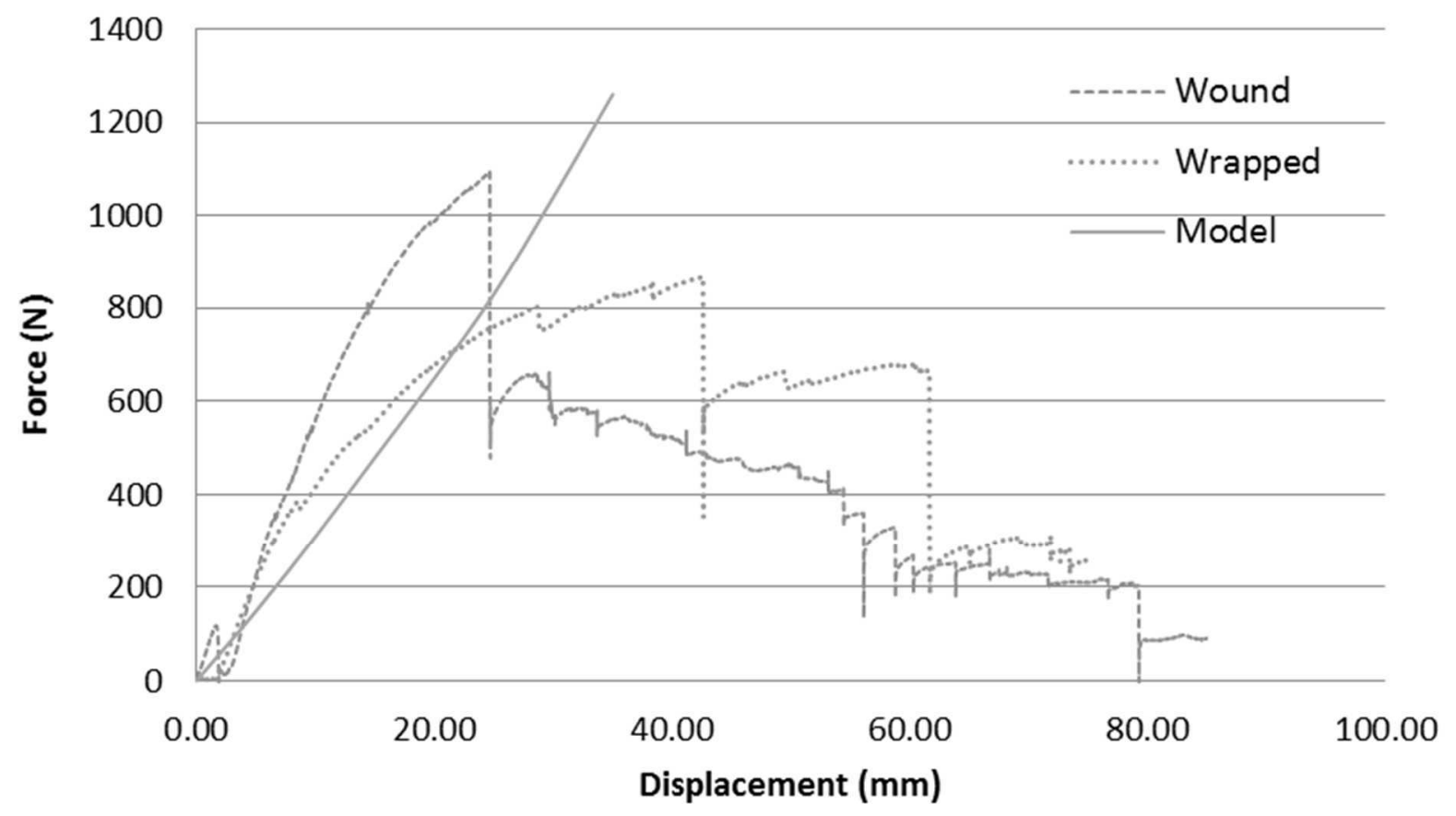

Fig. 7 The experimental and model results of the static bending test

In the pictures below are the results of the dynamic micro 3-points bending tests. In the Fig. 8 it is possible to see the mutual characteristic comparison (Fundamental and Loss modulus) of the two kinds of tubes in dependency on the loading frequency in the range $1-100 \mathrm{~Hz}$. 

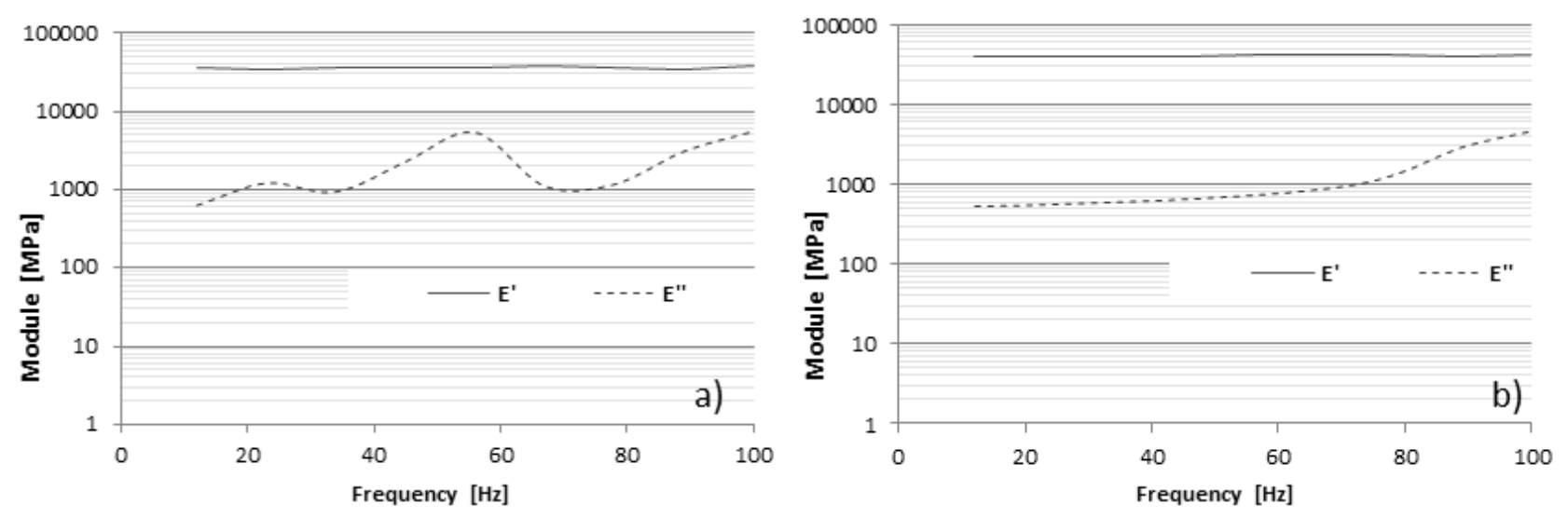

Fig. 8 The result of dynamic analysis a) Wrapped b) Wound, tube

\section{Conclusions}

Two sets of samples, one prepared by winding and second by wrapping technology of UD prepreg carbon materials were studied. Method one the wrapping of a wide tape had a very good surface quality and homogenity of material characteristics. In the second case to use nonconventional method of winding combined with pre-impregnated materials was tried. This method is suitable for straight shapes and with appropriate setting of the winding angle and can also create curved or closed shapes. A disadvantage was hat the fibers are often not ideally aligned as in the case of one wide tape and there are many places of their mutual overlapping which cause the resulting structure forming characteristic warp defects. What could be important is that the fibres are evidently more fastened. During the experiment we have obtained significantly different behavior of the two theoretically identical parts. There was not only different value of the maximal flexure strength, but also fundamentally different fracture mechanisms, delamination and the deformation of the entire test rods.

The found results have been evaluated also by using numerical model when the good conformity with the wrapped part was obtained in the beginning of loading. Then it the composite materials arose some issues like delamination or fractures. Therefore to our next work will be necessary to implement also the cohesive layers and damage mechanisms those could describe the arising material failures. In the term of numerical simulation of the wound parts, the entire approach to the model should be modified. In the first step is possible try to change the definition of input materials. It means do not use the verified material model of the ideal UD tape but homogenize the new tape by taking account the all occurring defects, pores and overlapping. However, it is also possible that the usual shell methods using stacked plies with different stiffness matrix will not be appropriate.

The main benefit of using prepreg instead of filaments is the simplification of consequent technologies like saturation and curing in form. As disadvantages are the considerably higher price of presaturated fibers and big, ideally constant preloading in the fibers throughout the entire manufacturing process. The winding technology has also a big potential to replace the nowadays insufficient wrapping methods.

What was quite interesting, although the wound structure is not so "arranged and aligned", it has higher overall stiffness in terms of stress and vibration transfer. However, it will be necessary to spend a long time by optimizing the final structure and also find the way, how to correctly simulate the material model, determine the mechanical behavior of this structure and also how to improve the technology in order to minimize all the imperfect places in the material structure.

\section{Acknowledgement}

This publication was written at the Technical University of Liberec as part of the project "Innovation of technical systems structures with the use of composite materials" with the support of the Specific University Research Grant, as provided by the Ministry of Education, Youth and Sports of the Czech Republic in the year 2018.

\section{References}

[1] HARRY, N. E., (2012). Clean filament winding: process optimization. United Kingdom, University Of Birmingham.

[2] KHERREDINE, L., GOUASMI, S., LAISSAOUI, R., ZEGHIB N. E. (2012). Evaluation and measurement of the damping properties of laminated CFRP composite plates. Materiaux, IOP Publishing, vol. 28.

[3] ZMINDAK, M., PELAGI, Z., SOUKUP, J. (2015). Analysis of Fiber Orientation Influence to Dynamic Properties of Composite Structures. $M a-$ nufacturing Technology, Vol. 15, No. 3. ISSN 1213-2489.

[4] SUGANUMA, Y., FUKUDA, H. (2007). Applicability of compression bending test to measure compressive failure strain, 16th conference on composite materials, Kyoto, Japan.

[5] KULHAVY, P., LEPSIK, P. (2018). Using Of Advanced Numerical Tools In Designing Composite 
Structures. Manufacturing technology, Vol. 18, No. 3. ISSN 1213-2489.

[6] ALLEN, A., K. (2004). A Method for Winding Advanced Composites of Unconventional Shapes using Continuous and Aligned Fibers, Brigham Young University.

[7] SEVCÍK, L., TUMA, P., PETRU, M., MARTINEC, T., KOVAR, R. (2013). Composite reinforcement, Patent Application no. 2013-1066.

[8] KOVAR, R., KULHAVY, P., LUFINKA, A. (2016). Stress analysis of the winding head frame for the production of the composites, 54th International conference on Experimental Stress Analysis, University of West Bohemia.

[9] ZHANG, P., SUN, R., ZHAO X., HU L. (2015). Placement suitability criteria of composite tape for mould surface in automated tape placement. Chinese Journal of Aeronautics, 28(5): 15741581.

[10] ULLAH, H., ET AL. (2012). Damage modelling in woven-fabric CFRP laminates under large-deflection bending. Computational Materials Science, Vol. 64.

[11] GRUBER, G., WARTZACK, S. (2013). Threepoint bending analyses of short fiber reinforced thermoplastic: Comparison between simulation and test results. SAS Tech Journal.
[12] ANSYS, Inc., (2010). Mechanical Structural Nonlinearities - Advanced Contact, Training Material.

[13] KIRSTEIN, A., F., WOOLLEY R., M. (1966). Symmetrical bending of thin circular elastic plates on equally spaced point supports. Washington DC: National Bureau of Standards.

[14] CHUNG, S., M., YAP, A., U., J., AND LIM, C., T. (2004). Flexural strength of dental composite restoratives, Comparison of biaxial and threepoint bending test, Journal of Biomed. Material Research.

[15] WEN, M., LUO, J. (2015): Study on Mechanical Properties of the Composite Resin Matrix Fiber Reinforced. Manufacturing Technology, Vol. 15, No. 2. ISSN 1213-2489.

[16] GRUBER, G., WARTZACK, S., (2013): Threepoint bending analyses of short fiber reinforced thermoplastic: Comparison between simulation and test results. SAS Tech. Journal.

[17] KULHAVY, P., LEPSIK, P. (2017). Study of bending properties of thin walled carbon and flax composite rods winded of prepreg fibers, $E A N$ 2017, Novy Smokovec, Slovakia.

[18] TA INSTRUMENTS - Waters LLC. (2006). QDMA Manual, New Castle, DE 19720. 